\title{
Efficacy of Combination Therapy With Pirfenidone and Low-Dose Cyclophosphamide for Refractory Interstitial Lung Disease Associated With Connective Tissue Disease: A Case-Series of Seven Patients
}

\author{
Lichong SHEN (D), Qingran YAN(D), Xiaoxiang CHEN(D) \\ Department of Rheumatology, Renji Hospital, School of Medicine, Shanghai Jiaotong University, Shanghai China
}

\begin{abstract}
Objectives: This study reports a low dose combination therapy of cyclophosphamide (CYC) and pirfenidone (PFD) and the efficiency and safety of the therapy in refractory connective tissue disease associated interstitial lung disease (CTD-ILD) patients.

Patients and methods: The study included seven CTD-ILD patients ( 2 males, 5 females; mean age 48.8 years; range, 32 to 63 years) treated between January 2016 and December 2017 in our clinic. At enrolment, all patients had shown no improvement in their symptoms (dyspnea or cough) after at least one month of high dose steroids treatment. Patients who had received adjusted immunosuppressive agents other than steroids or anti-fibrotic medications within the three months before enrolment were excluded. We changed the treatment to a low dose combination of $\mathrm{CYC} 0.4 \mathrm{~g} / \mathrm{m}^{2}$ monthly and PFD $300 \mathrm{mg}$ twice per day and quickly reduced the steroids. All the patients were followed-up for 12 months.

Results: Two patients had anti-synthetase syndrome, two had Sjögren syndrome, two had scleroderma and one had mixed connective tissue disease. The baseline forced vital capacity (FVC) was 39-81\% and the six-minute walk distance (6MWD) was $202 \mathrm{~m}-324 \mathrm{~m}$. Within 12 months followup, the median improvement in the FVC was $13.4 \%$ (range, 0-35.9\%), the median improvement of carbon monoxide diffusing capacity was $6.3 \%$ (range, 1.7-16\%) and the median improvement of 6MWD was 52.7\% (range, 34.4-86.3\%). All the patients were self-sufficient, and their dyspnea, chest high-resolution computed tomography scores, and quality of life improved simultaneously. Exceeding our expectations, no adverse events associated with CYC or PFD were observed during the follow-up period.

Conclusion: Our study provided preliminary while promising clinical evidence for combination therapy of CYC-PFD for CTD-ILD. A low dose combination of CYC and PFD was unexpectedly well tolerated, with satisfactory effects in refractory CTD-ILD patients. Well-designed controlled studies are needed to further establish the safety and efficacy of this approach.

Keywords: Cyclophosphamide, interstitial lung disease, pirfenidone.
\end{abstract}

Interstitial lung disease (ILD) is one of the most serious yet common conditions for patients with connective tissue diseases (CTDs), such as systemic sclerosis (SSc), ${ }^{1}$ dermatomyositis $(\mathrm{DM})^{2}$ and Sjögren syndrome (SS). ${ }^{3}$ CTD-ILD begins as an autoreactive inflammation and can progress to fibrosis. Either of these morbidities can cause lung dysfunction, greatly shorten survival and bring heavy disease burden to the patients.
To treat inflammation in CTD-ILD patients, one immunosuppressant plus steroids is a common regimen. In recent clinical trials on CTD-ILD, cyclophosphamide (CYC) is one of the most evidence-supported immunosuppressants, particularly for SSc-ILD. CYC improved the pulmonary function of 49.2 to $61.5 \%$ of the subjects during the first one year of follow-up, and improved dyspnea, high-resolution computed

Received: December 30, 2018 Accepted: May 16, 2018 Published online: August 26, 2019

Correspondence: Xiaoxiang Chen, MD. Renji Hospital, Shanghai Jiaotong University, School of Medicine, Rheumatology, 200025 Shanghai, China. Tel: 021-53882254 e-mail: chenxiaoxiang@renji.com 
tomography (HRCT) appearance and the healthrelated quality of life.4,5

To stop fibrosis, several small-molecule anti-fibrotic agents have been approved in recent decade. Pirfenidone (PFD) was the first of these agents. It is initially approved for idiopathic pulmonary fibrosis (IPF), the most treatmentresistant type of pulmonary fibrosis. In clinical trials on IPF, PFD improved patient forced vital capacity (FVC) and reduced the decline of the six-minute walk distance (6MWD). ${ }^{6,7}$ The application of PFD to CTD-ILD patients, including patients with SSc and DM, also showed acceptable efficacy, 8,9 despite most trials still being in progress.

To improve efficacy, a combination of an immunosuppressant and anti-fibrotic agent could be a reasonable approach. CYC is a traditional cytotoxic agent that kills activated lymphocytes to stop inflammation. On the other hand, PFD is an anti-fibrotic agent that inhibits collagen synthesis and fibroblast proliferation via pro-fibrotic growth factors. ${ }^{10,11}$ Based on their working mechanisms, PFD and CYC might work in synergy. In fact, PFD plus rapamycin have shown benefits in an alveolar cell line with pro-fibrotic stimulation. ${ }^{12}$

To our knowledge, thus far, the combination of PFD and CYC has never been reported, while other similar combinations have been assessed in two clinical trials of SSc-ILD: PFD with mycophenolate in Scleroderma Lung Study (SLS) III study (ClinicalTrials.gov: NCT03221257) and mycophenolate add on nintedanib in the Safety and Efficacy of Nintedanib in Systemic Sclerosis (SENSCIS) trial. ${ }^{13}$ The two studies are still in progress and both make use of full-dose combination.

A concern of full-dose combination is adverse effects. As monotherapy, pulse CYC at $1.0 \mathrm{~g} / \mathrm{m}^{2}$ caused nausea in $36.4 \%$ patients, hematuria in $13.6 \%$ patients and respiratory tract infections in $13.6 \%$ patients $^{14}$ while daily CYC can cause even higher incidence of side effects. ${ }^{4}$ When taking daily PFD up to $2400 \mathrm{mg}$, almost all patients suffered drug-associated adverse events such as rash or gastrointestinal intolerance. ${ }^{15}$ According to a meta-analysis of IPF trials, drug intolerance caused greater withdrawal of patients treated with PFD, compared with controls. ${ }^{16}$ Overall, a full-dose combination of
CYC and PFD might be risky for patients and hide potential benefits.

Therefore, we hypothesized that a low-dose combination may improve tolerance. Thus, in this study, we reported a low dose combination therapy of CYC and PFD and the efficiency and safety of the therapy in refractory CTD-ILD patients.

\section{PATIENTS AND METHODS}

Seven CTD-ILD patients (2 males, 5 females; mean age 48.8 years; range, 32 to 63 years) who had not shown improvement of their symptoms (dyspnea or cough) after at least one-month of steroid treatment (prednisone $\geq 1 \mathrm{mg} / \mathrm{kg}$ daily or were equivalent) were enrolled sequentially between January 2016 and December 2017, from Renji Hospital, Shanghai Jiao Tong University School of Medicine. Patients who had adjusted their immunosuppressive agents (including biological agents) other than steroids or had received anti-fibrotic medications within the three months before enrolment were excluded. Patients with dominant infection or other complications outside of the lungs that required daily prednisone over $1 \mathrm{mg} / \mathrm{kg}$ were also ruled out. The study protocol was approved by the Renji Hospital Ethics Committee. A written informed consent was obtained from each patient or his/her legal representative. The study was conducted in accordance with the principles of the Declaration of Helsinki.

We used the 6MWD to assess the patient activity tolerance ${ }^{17}$ and St. George's Respiratory Questionnaire (SGRQ) to evaluate quality of life. ${ }^{18}$ Pulmonary function tests (PFTs) and HRCT were performed at our hospital at three-six-month intervals, according to the changes of symptoms. The HRCT scores of the patients were calculated based on the Ichikado criterion by the same two radiologists. ${ }^{19}$

We generally changed the patients' previous treatments into a standard protocol consisting of intravenous CYC $0.4 \mathrm{~g} / \mathrm{m}^{2}$ per month and oral PFD $300 \mathrm{mg}$ twice daily. Additionally, we cut the oral steroids to prednisone $0.5 \mathrm{mg} / \mathrm{kg}$ daily (or equivalent) and tapered it to a stable dose (prednisone $\leq 10 \mathrm{mg}$ per day) within six months. 


\section{Statistical analysis}

All patients had HRCT scores, PFTs and 6MWD at baseline or follow-up to 12 months after changing their therapy. The differences in the median changes of diffusing capacity of lung for carbon monoxide (DLCO), FVC percentage, HRCT scores and 6MWD before and after treatment were assessed by Wilcoxon signed rank sum test. A $p$ value $<0.05$ was considered statistically significant.

\section{RESULTS}

Detailed demographic information is shown in Table 1. Two patients had anti-synthetase syndrome, two had SS, two had SSc and one had mixed connective tissue disease (MCTD). These patients were diagnosed as ILD by chest computed tomography (CT) two to five years before. At enrolment, the median 6MWD was $275 \mathrm{~m}$ (range, 202 to $324 \mathrm{~m}$ ), the median DLCO was $51 \%$ of the prediction (range, 47.7 to 63\%) and the median FVC was $72.3 \%$ of the prediction (range, 39 to $81 \%$ ). The median HRCT score was 150 (range, 136 to 203). All these patients had suffered severe dyspnea or cough, with the median baseline SGRQ score of 51 (range, 41 to 64).

After 12 months of CYC-PFD treatment, all patients showed significant improvement in their clinical status and HRCT. The PFTs were improved or stable. All patients who received steroids were successfully maintained at $10 \mathrm{mg}$ prednisone daily or less. The 6MWD showed a median increase of $52.7 \%$ (range, 34.4 to $86.3 \%$, $\mathrm{p}<0.05$ ). For quality of life assessment, the SGRQ total score showed a median improvement of $53.3 \%$ (range, 19.5 to $61.7 \%$, $\mathrm{p}<0.05)$. The HRCT score showed a median decrease of $20.1 \%$ (range, 11.7 to $29.6 \%$, $\mathrm{p}<0.05$ ). Regarding lung functions, the DLCO percentage showed a median improvement of $6.3 \%$ (range, 1.7 to $16 \%, \mathrm{p}<0.05$ ) and the FVC percentage showed a median improvement of $13.4 \%$ (range, 0 to $35.9 \%, \quad \mathrm{p}<0.05$ ) (Table 2, Figure 1a-f).

Although only seven patients were included in this study, it is worth mentioning that all patients tolerated the combination therapy unexpectedly well. During the 12-month follow-up, none of the patients had observable adverse events that could be attributed to PFD or CYC, such as gastrointestinal (nausea, dyspepsia, diarrhea, and anorexia), neurological (dizziness and fatigue), dermatological (photosensitivity, rash and pruritus), bone marrow suppression, hematuria, infection or menstrual disorders.

Patient 1: This patient is a 55-year-old male non-smoker, who was diagnosed with SS and ILD two years before. He was treated with oral methylprednisolone $8 \mathrm{mg}$ per day and hydroxychloroquine $0.2 \mathrm{~g}$ twice a day for at least one year. He had slowly progressive dyspnea for six months, but did not exhibit satisfactory improvement after one-month of $50 \mathrm{mg} /$ day

Table 1. Baseline characteristics of patients

\begin{tabular}{cccccc}
\hline Patient & Age (year)/Sex & ILD diagnosis (year) & Serum antibody & CTD diagnose* & $\begin{array}{c}\text { Baseline medication other than } \\
\text { steroids } \dagger\end{array}$ \\
\hline 1 & $55 / \mathrm{M}$ & 3 & Anti-SSA & SS & Hydroxychloroquine \\
2 & $43 / \mathrm{F}$ & 5 & Anti-OJ & ASS & Cyclosporine \\
3 & $41 / \mathrm{F}$ & 2 & Anti-U1RNP, Anti-SSA & MCTD & Methotrexate \\
4 & $53 / \mathrm{F}$ & 2.5 & Anti-Scl-70 & SSc & Hydroxychloroquine/colchicine \\
5 & $32 / \mathrm{F}$ & 3 & Anti-Scl-70 & SSc & Methotrexate \\
6 & $55 / \mathrm{M}$ & 2 & Anti-SSA & SS & Hydroxychloroquine \\
7 & $63 / \mathrm{F}$ & 2 & Anti-Jo-1 & ASS & Azathioprine \\
\hline
\end{tabular}

ILD: Interstitial lung disease; CTD: Connective tissue disease; SS: Sjögren syndrome; ASS: Anti-synthetase syndrome; MCTD: Mixed connective tissue disease; SSc: Systemic sclerosis; * Connective tissue disease diagnoses were established by consultant rheumatologists; $†$ These medications did not change during large dose steroids treatment (prednisone $\geq 1 \mathrm{mg} / \mathrm{kg}$ daily) before enrolment. 
Table 2. Parameters along with follow-up

\begin{tabular}{|c|c|c|c|c|c|}
\hline \multirow[t]{2}{*}{ Patients/parameter } & \multicolumn{2}{|c|}{ Pre-treatment } & \multicolumn{2}{|c|}{12 months post-treatment } & \multirow{2}{*}{$\begin{array}{c}\text { Change } \\
\%\end{array}$} \\
\hline & $\mathrm{n}$ & $\%$ & $\mathrm{n}$ & $\%$ & \\
\hline \multicolumn{6}{|l|}{ Patient 1} \\
\hline DLCO & & 50.4 & & 51.3 & +1.7 \\
\hline FVC & & 76.1 & & 88.4 & +16.2 \\
\hline 6MWD & 253 & & 488 & & +92.8 \\
\hline SGRQ total score $\dagger$ & 45 & & 21 & & -53.3 \\
\hline HRCT score§ & 146 & & 116 & & -20.5 \\
\hline \multicolumn{6}{|l|}{ Patient 2} \\
\hline DLCO & & 50 & & 58 & +16 \\
\hline FVC & & 39 & & 53 & +35.9 \\
\hline 6MWD & 202 & & 377 & & +86.3 \\
\hline SGRQ total score & 64 & & 36 & & -43.8 \\
\hline HRCT score & 203 & & 143 & & -29.6 \\
\hline \multicolumn{6}{|l|}{ Patient 3} \\
\hline DLCO & & 47.7 & & 53.2 & +11.5 \\
\hline FVC & & 72.3 & & 79.3 & +9.7 \\
\hline 6MWD & 279 & & 375 & & +34.4 \\
\hline SGRQ total score & 53 & & 31 & & -41.5 \\
\hline HRCT score & 143 & & 121 & & -15.4 \\
\hline \multicolumn{6}{|l|}{ Patient 4} \\
\hline DLCO & & 57.2 & & 60.3 & +5.4 \\
\hline FVC & & 62.6 & & 74.5 & +19 \\
\hline 6MWD & 275 & & 420 & & +52.7 \\
\hline SGRQ total score & 62 & & 25 & & -59.6 \\
\hline HRCT score & 150 & & 128 & & -14.6 \\
\hline \multicolumn{6}{|l|}{ Patient 5} \\
\hline DLCO & & 55 & & 57 & +3.6 \\
\hline FVC & & 81 & & 81 & 0 \\
\hline 6MWD & 324 & & 442.5 & & +36.5 \\
\hline SGRQ total score & 41 & & 33 & & -19.5 \\
\hline HRCT score & 136 & & 120 & & -11.7 \\
\hline \multicolumn{6}{|l|}{ Patient 6} \\
\hline DLCO & & 63 & & 67 & +6.3 \\
\hline FVC & & 67 & & 76 & +13.4 \\
\hline 6MWD & 267 & & 405 & & +46.7 \\
\hline SGRQ total score & 47 & & 18 & & -61.7 \\
\hline HRCT score & 169 & & 135 & & -20.1 \\
\hline \multicolumn{6}{|l|}{ Patient 7} \\
\hline DLCO & & 51 & & 58 & +13.7 \\
\hline FVC & & 76 & & 84 & +10.5 \\
\hline 6MWD & 274 & & 433 & & +58.0 \\
\hline SGRQ total score & 51 & & 23 & & -54.9 \\
\hline HRCT score & 176 & & 140 & & -20.5 \\
\hline
\end{tabular}


prednisone. At enrolment, he suffered an obvious aggravation in dyspnea and could only climb one flight of stairs. He had infiltration and fibrous cords as shown by HRCT. After two months of combined treatment, he was able to enjoy an overseas tour and could climb a hill over 200 meters high. His steroid treatment was changed to methylprednisolone $8 \mathrm{mg}$ per day at the seventh month of follow-up.

Patient 2: This patient is a 43-year-old female non-smoker with anti-synthetase antibodyassociated ILD. She experienced quickly worsening dyspnea and deteriorating PFTs over six weeks after being treated for four years with cyclosporine (75 mg twice a day) and prednisone (15 mg daily). She did not improve after increasing her steroid regimens (methylprednisolone $80 \mathrm{mg}$ for one week and prednisone $50 \mathrm{mg}$ for three weeks). She had resting dyspnea at enrolment. Her HRCT demonstrated the presence of infiltration and consolidation along the peripheral lung fields. After three months of combined treatment, she had no resting dyspnea. Twelve months later, her condition showed strikingly improved PFT with a 39.5\% improvement in FVC, with her prednisone dose maintained at $10 \mathrm{mg}$ per day.

Patient 3: This patient is a 41-year-old female non-smoker with MCTD-ILD. She was referred with rapidly progressive dyspnea within one month after being treated with methotrexate (MTX, $15 \mathrm{mg}$ per week) and prednisone (10 mg per day) for one year. After relapsing, she received $40 \mathrm{mg} /$ day prednisone but the progression of her symptoms did not stop. At enrolment, she had dyspnea when taking a shower or washing clothes. Her HRCT showed reticular fibrosis at the base of the lung. After six months of combined treatment, she had no difficulties with everyday housework or taking care of herself. Twelve months later, she could walk at least $1000 \mathrm{~m}$ on the ground without any rest or dyspnea, her prednisone dose maintained at $10 \mathrm{mg}$ for four months and $5 \mathrm{mg}$ for two months.

Patient 4: This patient is a 53-year-old female non-smoker diagnosed with SSc-ILD one-and-a-half years before. She had received hydroxychloroquine (0.2 g per day), colchicine (1 mg per day) and prednisone (10 mg per day) for over one year. She experienced progressive dyspnea for three months, although she had received $40 \mathrm{mg} /$ day prednisone in the last two months. At the time of enrolment, she could not even talk freely because of dyspnea. She had large scale of consolidation at the base of the lung. After 12 months of combined treatment, she could talk normally and walk on the ground for at least $500 \mathrm{~m}$ without dyspnea, with prednisone dose maintained at $10 \mathrm{mg}$ per day for six months.

Patient 5: This 32-year-old female nonsmoker was diagnosed with SSc-ILD and treated with MTX (10 mg per week) and prednisone (15 mg per day). For six months before enrolment, she had dry cough accompanied with chest pain when breathing. Her HRCT demonstrated diffused gross-glass opacity along peripheral lung fields. Because we first thought that MTX might be the reason, MTX was stopped and changed to prednisone $40 \mathrm{mg} /$ day for one month. Unfortunately, her symptoms were still aggravated and they bothered the patient in daily speaking and activity before enrolment. Her HRCT showed almost no change compared to one month earlier. Three months after the combination treatment, her cough and chest pain disappeared. After another 10-month follow-up, her PFTs remained stable, with an improved 6MWD and quality of life.

Patient 6: This patient is a 55-year-old male with more than 20-year smoking history (20 cigarettes a day on average). He was diagnosed as SS-ILD two years before, at which time he quit smoking. He was treated with prednisone (15 mg per day) and hydroxychloroquine (0.4 g per day) for over eight months. At enrolment, because of severe dyspnea, he had difficulty in talking for more than 10 minutes or climbing two flights of stairs because of dyspnea. Additionally, he had mild cough and felt severe fatigue all day. His chest HRCT showed diffused infiltration and reticular fibrosis, mostly along the peripheral area of the lungs (Figure 1f). After three months of combined treatment, he had no talking difficulties; 12 months later, he could climb five flights of stairs without dyspnea. His prednisone dose was maintained at $7.5 \mathrm{mg}$ per day since the ninth month of follow-up.

Patient 7: This patient is a 63-year-old female with a 15-year smoking history (approximately seven cigarettes per day) until she was diagnosed 
(a)

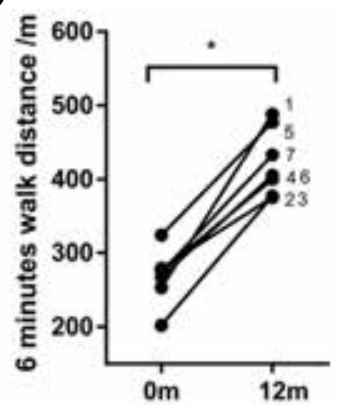

(c)

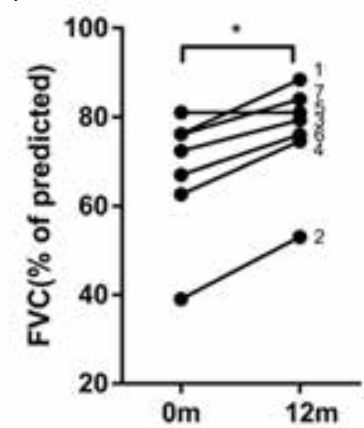

(e)

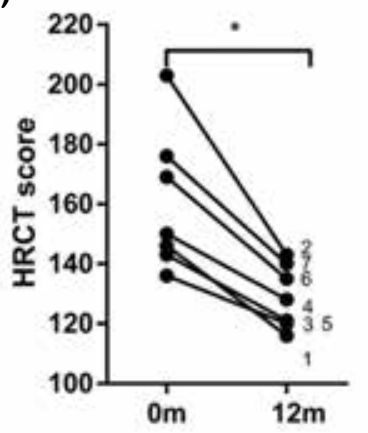

(b)

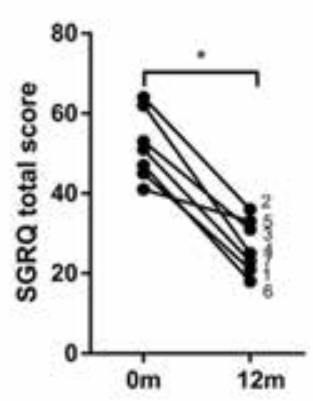

(d)

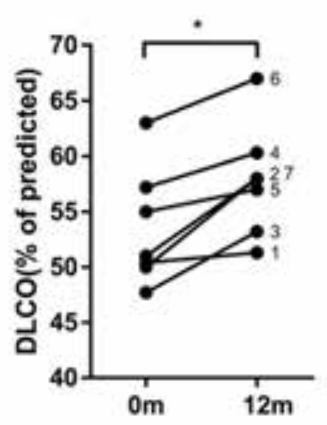

(f)

0
0
0
0
0
0
0
0
0
0
0
0
0

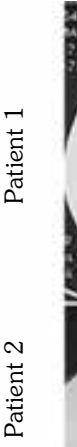

$0 \mathrm{M}$

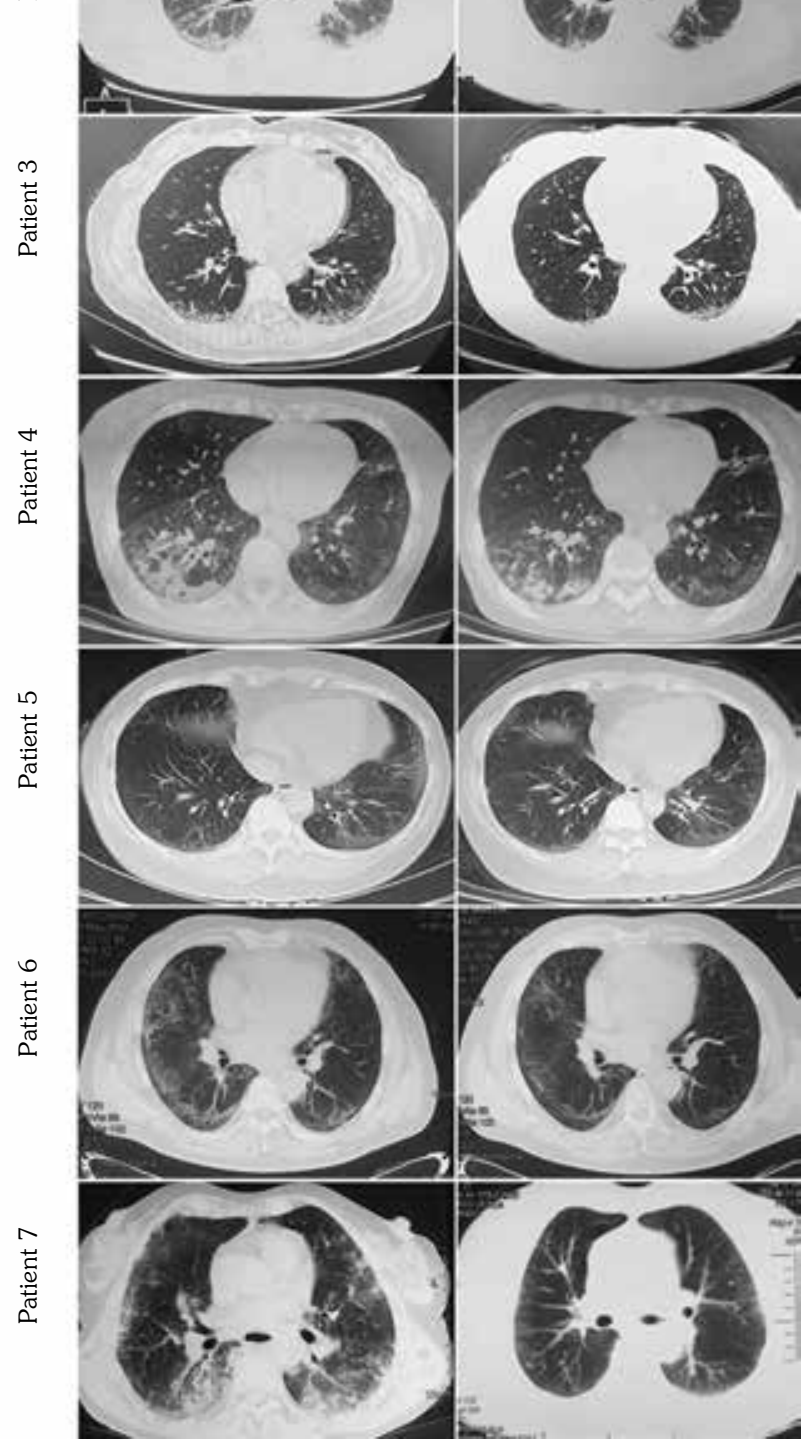

Figure 1. Parameters along with follow-up. (a) Patient activity tolerance was assessed by six-minute walk distance. (b) Patient dyspnea quality of life was assessed by St. George's Respiratory Questionnaire total score. (c and d) Pulmonary function tests, including forced vital capacity and diffusion capacity of lung for carbon monoxide. (e and f) High-resolution computed tomography scores and images. Various radiologic patterns, including infiltration, gross-glass opacity and fibrosis were found in seven patients. Numbers besides each point from picture A to E represent patient 1 to 7.

${ }^{*} \mathrm{P}<0.05$, Wilcoxon signed rank sum test.

SGRQ: St. George's Respiratory Questionnaire; FVC: Forced vital capacity; DLCO: Diffusion capacity of lung for carbon monoxide; HRCT: High-resolution computed tomography. 
as anti-synthetase antibody-associated ILD two years before. She was treated with azathioprine (50 mg per day) for one year. She developed dry cough three months prior to enrolment. She was first treated with $15 \mathrm{mg}$ /day prednisone for two months, but her symptoms still progressed. Her dose of prednisone was increased to $60 \mathrm{mg}$ per day for one month without additional improvement. At enrolment, she suffered dyspnea and dry cough, with difficulties in her daily housework. She had consolidation and ground glass opacity over almost $50 \%$ of her lung field (Figure 1f). After two months of combined treatment, she was not bothered by dyspnea or cough when doing housework or climbing three flights of stairs. After 12-month follow-up, her status remained stable with a prednisone dose at $7.5 \mathrm{mg}$ per day for six months.

\section{DISCUSSION}

The hypothesis that PFD and CYC might work in synergy is based on the different working mechanisms of these two agents. CYC is a classic alkylating agent that can induce cell death in resting and dividing lymphocytes and is highly potent in controlling a range of autoimmune diseases. ${ }^{20,21}$ PFD, a small molecular agent, can suppress the expression of growth factors, such as transforming growth factor-beta, fibroblast growth factor and platelet-derived growth factor, suppress proliferation of fibroblasts and inhibit collagen synthesis. Furthermore, it also inhibits monocyte secretion of inflammatory cytokines, such as tumor necrosis factor-alpha, interleukin (IL)-1 beta, and IL-6, and suppresses the production of reactive oxygen species in vivo and in vitro. ${ }^{10,11}$ Therefore, the combination of the two medicines may exhibit synergy in inhibition of the activation of inflammation and fibrosis as well as the progression of CTD-ILD.

Although the combination therapy is always an attractive idea, the likelihood of adverse effects is always a concern. In fact, there have been attempts to reduce the dose to improve the tolerance of either for CYC or PFD as monotherapy. MartinSuarez et al. ${ }^{22}$ first suggested reducing the CYC dose to $0.5 \mathrm{~g}$ per injection (first, $0.5 \mathrm{~g}$ weekly for three weeks and then $0.5 \mathrm{~g}$ monthly) to achieve less adverse effects. This regimen was verified in SSc-ILD patients by Paone et al. ${ }^{23}$ and Ando et al. ${ }^{24}$ who further reduced the dose to $0.5 \mathrm{~g}$ monthly, which is comparable to the dose of CYC selected in our combination treatment.

Compared with Caucasian patients, the adverse effect of PFD is a more prominent issue in East Asian patients. Because of its adverse effects, most clinical trials in East Asia chose $1800 \mathrm{mg} /$ day PFD, rather than the dose of $2400 \mathrm{mg} /$ day for American patients. Even so, the incidence of PFD related adverse events (such as photosensitivity, rash, gastrointestinal reaction, dizziness and fatigue) was over $50 \% .^{6,25}$ In a phase I trial for Chinese individuals, ${ }^{26}$ the incidence of adverse events was $20 \%$ at $600 \mathrm{mg} / \mathrm{day}$ PFD and rose up to $83 \%$ at $1200 \mathrm{mg} /$ day. Therefore, we chose $600 \mathrm{mg} /$ day PFD for the combination treatment to improve the tolerance.

In this cohort, all the patients unexpectedly tolerated the low dose combination therapy unexpectedly well. This approach might be feasible to improve the tolerance when treating CTD-ILD.

For low dose combination, insufficient efficacy is a potential concern. Although our study included a limited number of patients, our data show no apparent inferiority of the low dose combination therapy to CYC monotherapy. In this cohort, the median FVC and DLCO at baseline were $72 \%$ and $51 \%$ of the prediction, respectively, which appear to be similar to patients from the SLS I and II studies., ${ }^{4,5}$ In these clinical trials, approximately $20-30 \%$ patients from the CYC group had decreased FVC/DLCO at the end of the follow-up, while in our study, three/seven patients had elevated predicted FVC $\geq 15 \%$ or predicted DLCO $\geq 10 \%$, six/seven patients had improved FVC, seven/seven patients had improved DLCO and no patient exhibited deterioration in PFTs after one-year of treatment. Our data for the first time suggest that the low dose combination of PFD and CYC warrants further investigation.

One confounder of this observation is the use of steroids before the enrolment. We could not rule out the effects of steroids. However, at enrolment, we had to step-up the treatment without washing-out as clinical practitioners. According to reports on ILD associated with DM or polymyositis, ${ }^{27,28}$ once a patient began treatment with high dose steroids, if the respiratory 
symptoms were not improved within four weeks, the survival would be poor (less than $40 \%$ in 10 weeks after the treatment) if the patient did not promptly receive immunosuppressants. Until now, no strong evidence has demonstrated that ILD associated with most CTDs has a substantially different mode of progression. The only known exception is the anti-melanoma differentiationassociate gene (anti-MDA5) antibody associated rapidly progressing ILD, ${ }^{29}$ which is not included in this study. Given the condition of our patients, although not all the patients had myositis, we thought waiting or washing-out would be relatively unsafe. And the concomitant steroids thus became an inevitable major confounder. Full-dose controlled clinical trials with longer observation are needed with restriction on the use of steroids to better assess the low dose combination therapy.

In conclusion, our study provided preliminary but promising clinical evidence for combination therapy of CYC and PFD for CTD-ILD. A low dose combination of CYC and PFD was well tolerated, with satisfactory effects in refractory CTD-ILD patients. Well-designed controlled studies are needed to further establish the safety and efficacy of this approach.

\section{Declaration of conflicting interests}

The authors declared no conflicts of interest with respect to the authorship and/or publication of this article.

\section{Funding}

This work was supported by the National Natural Science Foundation of China (No. 81601401 and No. 81771733).

\section{REFERENCES}

1. Jung E, Suh $\mathrm{CH}$, Kim HA, Jung JY. Clinical Characteristics of Systemic Sclerosis With Interstitial Lung Disease. Arch Rheumatol 2018;33:322-7.

2. Rozman B, Bozic B, Kveder T. Interstitial lung disease, a common manifestation of newly diagnosed polymyositis and dermatomyositis. Ann Rheum Dis 2004;63:1005.

3. Yazisiz V, Arslan G, Ozbudak IH, Turker S, Erbasan $\mathrm{F}$, Avci $\mathrm{AB}$, et al. Lung involvement in patients with primary Sjögren's syndrome: what are the predictors? Rheumatol Int 2010;30:1317-24.

4. Tashkin DP, Elashoff R, Clements PJ, Goldin J, Roth MD, Furst DE, et al. Cyclophosphamide versus placebo in scleroderma lung disease. N Engl J Med 2006;354:2655-66.

5. Tashkin DP, Roth MD, Clements PJ, Furst DE, Khanna D, Kleerup EC, et al. Mycophenolate mofetil versus oral cyclophosphamide in sclerodermarelated interstitial lung disease (SLS II): a randomised controlled, double-blind, parallel group trial. Lancet Respir Med 2016;4:708-19.

6. Azuma A, Nukiwa T, Tsuboi E, Suga M, Abe S, Nakata K, et al. Double-blind, placebo-controlled trial of pirfenidone in patients with idiopathic pulmonary fibrosis. Am J Respir Crit Care Med 2005;171:1040-7.

7. King TE Jr, Bradford WZ, Castro-Bernardini S, Fagan EA, Glaspole I, Glassberg MK, et al. A phase 3 trial of pirfenidone in patients with idiopathic pulmonary fibrosis. N Engl J Med 2014;370:2083-92.

8. Li T, Guo L, Chen Z, Gu L, Sun F, Tan X, et al. Pirfenidone in patients with rapidly progressive interstitial lung disease associated with clinically amyopathic dermatomyositis. Sci Rep 2016;6:33226.

9. Miura Y, Saito T, Fujita K, Tsunoda Y, Tanaka T, Takoi $\mathrm{H}$, et al. Clinical experience with pirfenidone in five patients with scleroderma-related interstitial lung disease. Sarcoidosis Vasc Diffuse Lung Dis 2014;31:235-8.

10. Macías-Barragán J, Sandoval-Rodríguez A, NavarroPartida J, Armendáriz-Borunda J. The multifaceted role of pirfenidone and its novel targets. Fibrogenesis Tissue Repair 2010;3:16.

11. Oku H, Shimizu T, Kawabata T, Nagira M, Hikita I, Ueyama A, et al. Antifibrotic action of pirfenidone and prednisolone: different effects on pulmonary cytokines and growth factors in bleomycin-induced murine pulmonary fibrosis. Eur J Pharmacol 2008;590:400-8.

12. Molina-Molina M, Machahua-Huamani C, VicensZygmunt V, Llatjós R, Escobar I, Sala-Llinas E, et al. Anti-fibrotic effects of pirfenidone and rapamycin in primary IPF fibroblasts and human alveolar epithelial cells. BMC Pulm Med 2018;18:63.

13. Distler O, Brown KK, Distler JHW, Assassi S, Maher TM, Cottin V, et al. Design of a randomised, placebocontrolled clinical trial of nintedanib in patients with systemic sclerosis-associated interstitial lung disease (SENSCISTM). Clin Exp Rheumatol 2017;35:75-81.

14. Hoyles RK, Ellis RW, Wellsbury J, Lees B, Newlands P, Goh NS, et al. A multicenter, prospective, randomized, double-blind, placebo-controlled trial of corticosteroids and intravenous cyclophosphamide followed by oral azathioprine for the treatment of pulmonary fibrosis in scleroderma. Arthritis Rheum 2006;54:3962-70.

15. Lancaster LH, de Andrade JA, Zibrak JD, Padilla ML, Albera C, Nathan SD, et al. Pirfenidone safety and adverse event management in idiopathic pulmonary fibrosis. Eur Respir Rev 2017;26(146). pii: 170057.

16. Jiang $C$, Huang $H$, Liu J, Wang $Y$, Lu Z, Xu Z. Adverse events of pirfenidone for the treatment of pulmonary fibrosis: a meta-analysis of randomized controlled trials. PLoS One 2012;7:e47024. 
17. ATS Committee on Proficiency Standards for Clinical Pulmonary Function Laboratories. ATS statement: guidelines for the six-minute walk test. Am J Respir Crit Care Med 2002;166:111-7.

18. Suzuki A, Kondoh Y, Swigris JJ, Ando M, Kimura $\mathrm{T}$, Kataoka K, et al. Performance of the St George's Respiratory Questionnaire in patients with connective tissue disease-associated interstitial lung disease. Respirology 2018 Mar 25.

19. Ichikado K, Suga M, Muranaka H, Gushima Y, Miyakawa $\mathrm{H}$, Tsubamoto $\mathrm{M}$, et al. Prediction of prognosis for acute respiratory distress syndrome with thin-section CT: validation in 44 cases. Radiology 2006;238:321-9.

20. Novack SN, Pearson CM. Cyclophosphamide therapy in Wegener's granulomatosis. N Engl J Med 1971;284:938-42.

21. Steinberg AD, Steinberg SC. Long-term preservation of renal function in patients with lupus nephritis receiving treatment that includes cyclophosphamide versus those treated with prednisone only. Arthritis Rheum 1991;34:945-50.

22. Martin-Suarez I, D'Cruz D, Mansoor M, Fernandes AP, Khamashta MA, Hughes GR. Immunosuppressive treatment in severe connective tissue diseases: effects of low dose intravenous cyclophosphamide. Ann Rheum Dis 1997;56:481-7.

23. Paone C, Chiarolanza I, Cuomo G, Ruocco L, Vettori $\mathrm{S}$, Menegozzo $\mathrm{M}$, et al. Twelve-month azathioprine as maintenance therapy in early diffuse systemic sclerosis patients treated for 1-year with low dose cyclophosphamide pulse therapy. Clin Exp Rheumatol 2007;25:613-6.

24. Ando K, Motojima S, Doi T, Nagaoka T, Kaneko $\mathrm{N}$, Aoshima $\mathrm{M}$, et al. Effect of glucocorticoid monotherapy on pulmonary function and survival in Japanese patients with scleroderma-related interstitial lung disease. Respir Investig 2013;51:69-75.

25. Huang H, Dai HP, Kang J, Chen BY, Sun TY, Xu ZJ. Double-blind randomized trial of pirfenidone in Chinese idiopathic pulmonary fibrosis patients. medicine (Baltimore) 2015;94:e1600.

26. Huang NY, Ding L, Wang J, Zhang QY, Liu X, Lin $\mathrm{HD}$, et al. Pharmacokinetics, safety and tolerability of pirfenidone and its major metabolite after single and multiple oral doses in healthy Chinese subjects under fed conditions. Drug Res (Stuttg) 2013;63:388-95.

27. Nawata Y, Kurasawa K, Takabayashi K, Miike $\mathrm{S}$, Watanabe $\mathrm{N}$, Hiraguri $\mathrm{M}$, et al. Corticosteroid resistant interstitial pneumonitis in dermatomyositis/ polymyositis: prediction and treatment with cyclosporine. J Rheumatol 1999;26:1527-33.

28. Kurasawa K, Nawata Y, Takabayashi K, Kumano K, Kita Y, Takiguchi Y, et al. Activation of pulmonary T cells in corticosteroid-resistant and -sensitive interstitial pneumonitis in dermatomyositis/polymyositis. Clin Exp Immunol 2002;129:541-8.

29. Chen F, Wang D, Shu X, Nakashima R, Wang G. Anti-MDA5 antibody is associated with A/SIP and decreased $\mathrm{T}$ cells in peripheral blood and predicts poor prognosis of ILD in Chinese patients with dermatomyositis. Rheumatol Int 2012;32:3909-15. 